\title{
The Crack Tip Fields for Anti-plane Crack in Functionally Graded Magnetoelectroelastic Materials
}

\author{
Guangtu SHEN, Xiao CHONG, Peng ZHANG \\ School of Noncommissioned Officer \\ The University of Astronautics engineering \\ Beijing, China \\ e-mail: chongxiao2005@163.com
}

\author{
Yao DAI \\ The department of mechanical engineering \\ The Academy of Armored Force Engineering \\ Beijing, China \\ e-mail: dai_yao@sina.com
}

\begin{abstract}
The problem of a anti-plane crack in functionally graded magnetoelectroelastic materials is investigated. The material properties of the functionally graded magnetoelectroelastic materials are assumed to be exponential function of $y$ perpendicular to the crack. To make the analysis tractable, the crack surface condition is assumed to be electrically and magnetically impermeable. The high order crack tip fields are obtained by the method of eigen-expansion method. This study has fundamental significance as Williams' solution.
\end{abstract}

Keywords-component; crack tip fields; eigen-expansion method; Williams' solution; functionally graded magnetoelectroelastic materials

\section{INTRODUCTION}

Due to magnetoelectroelastic coupling behavior, the composite materials are extensively used to design actuators, sensors and other electronic products in modern technology, particularly in smart materials/intelligent structures that are cable of responding to internal and environment changes. With the applications of smart devices such as actuators, sensors and other adaptive control units in aeronautics, astronautics, communication, biomedical industry and so on, the research on the smart materials in these devices has been receiving increasing attentions nowadays. However, under the mechanical and/or electric and/or magnetic loading, these materials are usually predisposed to fracture owing to the existing microscopic defects. In recent years, an area of increasing interest is the fracture mechanics of magnetoelectroelastic materials, which combine the ferromagnetic and ferroelectric phases. For inclusions and cracks embedded in such magnetoelectroelastic solids, magnetoelectroelastic behaviors have been analyzed by many researchers including Wang et al. [1], Wan et al. [2],Zhong and Li [3], Huang et al. [4], Gao et al.[5], Sih et al. [6], Li and Lee [7], Athanasius and Ang [8], Bhargava and Sharma [9], Tupholme [10], Wang and Mai [11], Zheng et al. [12], Ma et al. [13] and Li et al. [14] . It can be found that only the crack tip singular field is involved in the researches mentioned above. This is because that the eigen-functions or the higherorder crack tip fields of the functionally graded magnetoelectroelastic materials as Williams' solutions have not been available upto now. Therefore, the main effort of this paper is to find out the higher-order crack tip fields of the crack of functionally graded magnetoelectroelastic materials by the eigen-expansion method.

\section{BASIC EQUATIONS}

Consider the functionally graded magnetoelectroelastic material containing a crack. For anti-plane problems, only the out-of-plane displacement, the in-plane electric fields and the in-plane magnetic fields are of interest. Therefore, only the anti-plane deformation is coupled with the in-plane magnetoelectric fields, and the constitutive relation of the magnetoelectroelastic material to reduced to

$$
\boldsymbol{\tau}_{r}=\mathbf{M} \frac{\partial \mathbf{w}}{\partial r} \quad \boldsymbol{\tau}_{\theta}=\mathbf{M} \frac{\partial \mathbf{w}}{r \partial \theta}
$$

where $\tau_{k}=\left[\begin{array}{lll}\tau_{k z} & D_{k} & B_{k}\end{array}\right]^{\mathrm{T}} \quad(k=r, \theta)$ is the generalized stress and $\tau, D, B$ are the anti-plane stress, electric displacement, magnetic induction, respectively. $\mathbf{w}=\left[\begin{array}{lll}w & \phi & \varphi\end{array}\right]^{\mathrm{T}}$ is the generalized displacement and $w, \phi, \varphi$ are the anti-plane mechanical displacement, electric potential, magnetic potential, respectively.

The property matrix

$$
\mathbf{M}=\left[\begin{array}{ccc}
c_{44} & e_{15} & h_{15} \\
e_{15} & -\varepsilon_{11} & -d_{11} \\
h_{15} & -d_{11} & -\mu_{11}
\end{array}\right]
$$

where $c_{44}, e_{15}, h_{15}, \varepsilon_{11}, d_{11}, \mu_{11}$ are the shear modulus, piezoelectric coefficient, piezomagnetic coefficient, dielectric coefficient, magnetoelectric coefficient, and magnetic permeability, respectively. The present work employs exponential functions to describe the continuous variations of material properties,

$$
\begin{gathered}
\mathbf{M}=\mathbf{M}_{0} e^{\beta r \sin \theta} \\
\mathbf{M}_{0}=\left[\begin{array}{ccc}
c_{440} & e_{150} & h_{150} \\
e_{150} & -\varepsilon_{110} & -d_{110} \\
h_{150} & -d_{110} & -\mu_{110}
\end{array}\right]
\end{gathered}
$$


where $\quad c_{4}{ }_{4},{ }_{0} e{ }_{1},{ }_{5} h_{0} \varepsilon \quad{ }_{5}{ }_{0}$, and $\mu_{110}$ are the shear modulus, piezoelectric coefficient, piezomagnetic coefficient, dielectric coefficient, magnetoelectric coefficient, and magnetic permeability at $y=0$.

The generalized equilibrium equations can be written as

$$
\boldsymbol{\tau}_{r, r}+\frac{1}{r} \boldsymbol{\tau}_{\theta, \theta}+\frac{1}{r} \boldsymbol{\tau}_{r}=0
$$

Substituting (1) and (2) into (5) yields the governing equations

$$
\mathbf{M} \nabla^{2} \mathbf{w}+\frac{\partial \mathbf{M}}{\partial r} \frac{\partial \mathbf{w}}{\partial r}+\frac{1}{r^{2}} \frac{\partial \mathbf{M}}{\partial \theta} \frac{\partial \mathbf{w}}{\partial \theta}=0
$$

where $\nabla^{2}=\frac{\partial^{2}}{\partial r^{2}}+\frac{1}{r} \frac{\partial}{\partial r}+\frac{1}{r^{2}} \frac{\partial^{2}}{\partial \theta^{2}}$ is the two-dimensional Laplace operator.

\section{THE HIGHER ORDER CRACK-TIP FIELDS}

The displacement component $w$, electric potential $\phi$ and magnetic potential $\varphi$ can be expanded as follows

$$
\mathbf{w}=\sum_{n=1}^{\infty} r^{\frac{n}{2}} \mathbf{w}_{n}(\theta)
$$

where, $\mathbf{w}_{n}(\theta)=\left[\begin{array}{lll}w_{n}(\theta) & \phi_{n}(\theta) & \varphi_{n}(\theta)\end{array}\right]^{\mathrm{T}}$ and $w_{n}(\theta), \phi_{n}(\theta)$, $\varphi_{n}(\theta)$ are eigen-functions.

Substitute (7) into (6). According to the linear independence of $r^{-3 / 2}, r^{-1}, r^{-1 / 2}, \ldots, r^{i / 2-2}, \ldots$, the eigenequations are obtained. The system of ordinary differential equations are

$$
\left\{\begin{array}{l}
\frac{1}{4} \frac{\mathrm{d} \mathbf{w}_{1}(\theta)}{\mathrm{d} \theta}+\frac{\mathrm{d}^{2} \mathbf{w}_{1}(\theta)}{\mathrm{d} \theta^{2}}=0 \\
\mathbf{w}_{2}(\theta)+\frac{\mathrm{d}^{2} \mathbf{w}_{2}(\theta)}{\mathrm{d} \theta^{2}}=0 \\
\frac{9}{4} \mathbf{w}_{3}(\theta)+\frac{\mathrm{d}^{2} \mathbf{w}_{3}(\theta)}{\mathrm{d} \theta^{2}}+\frac{1}{2} \beta \sin \theta \mathbf{w}_{1}(\theta)+\beta \cos \theta \frac{\mathrm{d} \mathbf{w}_{1}(\theta)}{\mathrm{d} \theta}=0 \\
4 \mathbf{w}_{4}(\theta)+\frac{\mathrm{d}^{2} \mathbf{w}_{4}(\theta)}{\mathrm{d} \theta^{2}}+\beta \sin \theta \mathbf{w}_{2}(\theta)+\beta \cos \theta \frac{\mathrm{d} \mathbf{w}_{2}(\theta)}{\mathrm{d} \theta}=0 \\
\frac{25}{4} \mathbf{w}_{5}(\theta)+\frac{\mathrm{d}^{2} \mathbf{w}_{5}(\theta)}{\mathrm{d} \theta^{2}}+\frac{3}{2} \beta \sin \theta \mathbf{w}_{3}(\theta)+\beta \cos \theta \frac{\mathrm{d} \mathbf{w}_{3}(\theta)}{\mathrm{d} \theta}=0 \\
9 \mathbf{w}_{6}(\theta)+\frac{\mathrm{d}^{2} \mathbf{w}_{6}(\theta)}{\mathrm{d} \theta^{2}}+2 \beta \sin \theta \mathbf{w}_{4}(\theta)+\beta \cos \theta \frac{\mathrm{d} \mathbf{w}_{4}(\theta)}{\mathrm{d} \theta}=0 \\
\cdots \ldots . .
\end{array}\right.
$$

In the case of electrically and magnetically impermeable crack, the boundary conditions are

$$
\left.\boldsymbol{\tau}_{r}\right|_{\theta= \pm \pi}=0
$$

Further, they can be expressed as

$$
\left.\frac{d \mathbf{w}_{n}(\theta)}{d \theta}\right|_{\theta= \pm \pi}=0
$$

Solving the system of ordinary differential equations, we can obtain the results

$$
\left\{\begin{array}{l}
\mathbf{w}_{1}(\theta)=\mathbf{A}_{11} \sin \frac{\theta}{2} \\
\mathbf{w}_{2}(\theta)=\mathbf{A}_{21} \cos \theta \\
\mathbf{w}_{3}(\theta)=\mathbf{A}_{31} \sin \frac{3 \theta}{2}-\frac{1}{12} \beta \mathbf{A}_{11} \cos \frac{3 \theta}{2}-\frac{1}{4} \beta \mathbf{A}_{11} \cos \frac{\theta}{2} \\
\mathbf{w}_{4}(\theta)=\mathbf{A}_{41} \cos 2 \theta \\
\mathbf{w}_{5}(\theta)=\mathbf{A}_{51} \sin \frac{5 \theta}{2}+\frac{1}{20} \beta \mathbf{A}_{31} \cos \frac{5 \theta}{2}+\frac{1}{32} \beta^{2} \mathbf{A}_{11} \sin \frac{3 \theta}{2}+ \\
\quad \frac{1}{48} \beta^{2} \mathbf{A}_{11} \sin \frac{\theta}{2}-\frac{1}{4} \beta \mathbf{A}_{31} \cos \frac{\theta}{2} \\
\mathbf{w}_{6}(\theta)=\mathbf{A}_{61} \cos 3 \theta-\frac{1}{12} \beta \mathbf{A}_{41} \sin 3 \theta+\frac{1}{4} \beta \mathbf{A}_{41} \cos 3 \theta \\
\ldots \ldots .
\end{array}\right.
$$

where $\mathbf{A}_{i j}=\left[\begin{array}{lll}A_{i j} & B_{i j} & C_{i j}\end{array}\right]$ and $A_{i j}, B_{i j}, C_{i j}$ are the undetermined coefficients.

Substituting (11) into (7), the generalized displacement is obtained.

$$
\begin{aligned}
\mathbf{w}= & r^{\frac{1}{2}} \mathbf{A}_{11} \sin \frac{\theta}{2}+r \mathbf{A}_{21} \cos \theta+r^{\frac{3}{2}}\left(\mathbf{A}_{31} \sin \frac{3 \theta}{2}-\frac{1}{12} \beta \mathbf{A}_{11} .\right. \\
& \left.\cos \frac{3 \theta}{2}-\frac{1}{4} \beta \mathbf{A}_{11} \cos \frac{\theta}{2}\right)+r^{2} \mathbf{A}_{41} \cos 2 \theta+r^{\frac{5}{2}}\left(\mathbf{A}_{51} .\right. \\
& \sin \frac{5 \theta}{2}+\frac{1}{20} \beta \mathbf{A}_{31} \cos \frac{5 \theta}{2}+\frac{1}{32} \beta^{2} \mathbf{A}_{11} \sin \frac{3 \theta}{2}+ \\
& \left.\frac{1}{48} \beta^{2} \mathbf{A}_{11} \sin \frac{\theta}{2}-\frac{1}{4} \beta \mathbf{A}_{31} \cos \frac{\theta}{2}\right)+r^{3}\left(\mathbf{A}_{61} \cos 3 \theta-\right. \\
& \left.\frac{1}{12} \beta \mathbf{A}_{41} \sin 3 \theta+\frac{1}{4} \beta \mathbf{A}_{41} \cos 3 \theta\right)+\cdots
\end{aligned}
$$

Then, the stress, electric displacement and magnetic induction components can be obtained 


$$
\begin{aligned}
& \tau_{r z}=c_{44}\left[\frac{1}{2} r^{-\frac{1}{2}} A_{11} \sin \frac{\theta}{2}+A_{21} \cos \theta+\frac{3}{2} r^{\frac{1}{2}}\left(A_{31} \sin \frac{3 \theta}{2}-\right.\right. \\
& \left.\frac{1}{12} \beta A_{11} \cos \frac{3 \theta}{2}-\frac{1}{4} \beta A_{11} \cos \frac{\theta}{2}\right)+2 r A_{41} \cos 2 \theta+ \\
& \frac{5}{2} r^{\frac{3}{2}}\left(A_{51} \sin \frac{5 \theta}{2}+\frac{1}{20} \beta A_{31} \cos \frac{5 \theta}{2}+\frac{1}{32} \beta^{2} A_{11} \sin \frac{3 \theta}{2}+\right. \\
& \left.\frac{1}{48} \beta^{2} A_{11} \sin \frac{\theta}{2}-\frac{1}{4} \beta A_{31} \cos \frac{\theta}{2}\right)+3 r^{2}\left(A_{61} \cos 3 \theta-\right. \\
& \left.\left.\frac{1}{12} \beta A_{41} \sin 3 \theta+\frac{1}{4} \beta A_{41} \cos 3 \theta\right)\right]+e_{15}\left[\frac{1}{2} r^{-\frac{1}{2}} B_{11} \sin \frac{\theta}{2}+\right. \\
& B_{21} \cos \theta+\frac{3}{2} r^{\frac{1}{2}}\left(B_{31} \sin \frac{3 \theta}{2}-\frac{1}{12} \beta B_{11} \cos \frac{3 \theta}{2}-\frac{1}{4} \beta .\right. \\
& \left.B_{11} \cos \frac{\theta}{2}\right)+2 r B_{41} \cos 2 \theta+\frac{5}{2} r^{\frac{3}{2}}\left(B_{51} \sin \frac{5 \theta}{2}+\frac{1}{20} \beta\right. \text {. } \\
& B_{31} \cos \frac{5 \theta}{2}+\frac{1}{32} \beta^{2} B_{11} \sin \frac{3 \theta}{2}+\frac{1}{48} \beta^{2} B_{11} \sin \frac{\theta}{2}- \\
& \left.\frac{1}{4} \beta B_{31} \cos \frac{\theta}{2}\right)+3 r^{2}\left(B_{61} \cos 3 \theta-\frac{1}{12} \beta B_{41} \sin 3 \theta+\right. \\
& \left.\left.\frac{1}{4} \beta B_{41} \cos 3 \theta\right)\right]+h_{15}\left[\frac{1}{2} r^{-\frac{1}{2}} C_{11} \sin \frac{\theta}{2}+C_{21} \cos \theta+\right. \\
& \frac{3}{2} r^{\frac{1}{2}}\left(C_{31} \sin \frac{3 \theta}{2}-\frac{1}{12} \beta C_{11} \cos \frac{3 \theta}{2}-\frac{1}{4} \beta C_{11} \cos \frac{\theta}{2}\right)+ \\
& 2 r C_{41} \cos 2 \theta+\frac{5}{2} r^{\frac{3}{2}}\left(C_{51} \sin \frac{5 \theta}{2}+\frac{1}{20} \beta C_{31} \cos \frac{5 \theta}{2}+\right. \\
& \left.\frac{1}{32} \beta^{2} C_{11} \sin \frac{3 \theta}{2}+\frac{1}{48} \beta^{2} C_{11} \sin \frac{\theta}{2}-\frac{1}{4} \beta C_{31} \cos \frac{\theta}{2}\right)+ \\
& \left.3 r^{2}\left(C_{61} \cos 3 \theta-\frac{1}{12} \beta C_{41} \sin 3 \theta+\frac{1}{4} \beta C_{41} \cos 3 \theta\right)\right]
\end{aligned}
$$$$
\tau_{\theta z}=c_{44}\left[\frac{1}{2} r^{-\frac{1}{2}} A_{11} \cos \frac{\theta}{2}-A_{21} \sin \theta+r^{\frac{1}{2}}\left(\frac{3}{2} A_{31} \cos \frac{3 \theta}{2}+\right.\right.
$$$$
\left.\frac{1}{8} \beta A_{11} \sin \frac{3 \theta}{2}+\frac{1}{8} \beta A_{11} \sin \frac{\theta}{2}\right)-r A_{41} \sin 2 \theta+r^{\frac{3}{2}} \text {. }
$$$$
\left(\frac{5}{2} A_{51} \cos \frac{5 \theta}{2}-\frac{1}{8} \beta A_{31} \sin \frac{5 \theta}{2}+\frac{3}{64} \beta^{2} A_{11} \cos \frac{3 \theta}{2}+\right.
$$$$
\left.\frac{1}{96} \beta^{2} A_{11} \cos \frac{\theta}{2}+\frac{1}{8} \beta A_{31} \sin \frac{\theta}{2}\right)-r^{2}\left(3 A_{61} \sin 3 \theta+\right.
$$$$
\left.\left.\frac{1}{4} \beta A_{41} \cos 3 \theta+\frac{3}{4} \beta A_{41} \sin 3 \theta\right)\right]+e_{15}\left[\frac{1}{2} r^{-\frac{1}{2}} B_{11}\right. \text {. }
$$$$
\cos \frac{\theta}{2}-B_{21} \sin \theta+r^{\frac{1}{2}}\left(\frac{3}{2} B_{31} \cos \frac{3 \theta}{2}+\frac{1}{8} \beta B_{11}\right.
$$$$
\left.\sin \frac{3 \theta}{2}+\frac{1}{8} \beta B_{11} \sin \frac{\theta}{2}\right)-r B_{41} \sin 2 \theta+r^{\frac{3}{2}}\left(\frac{5}{2} B_{51}\right. \text {. }
$$$$
\cos \frac{5 \theta}{2}-\frac{1}{8} \beta B_{31} \sin \frac{5 \theta}{2}+\frac{3}{64} \beta^{2} B_{11} \cos \frac{3 \theta}{2}+
$$$$
\left.\frac{1}{96} \beta^{2} B_{11} \cos \frac{\theta}{2}+\frac{1}{8} \beta B_{31} \sin \frac{\theta}{2}\right)-r^{2}\left(3 B_{61} \sin 3 \theta+\right.
$$

$$
\begin{aligned}
& \left.\left.\frac{1}{4} \beta B_{41} \cos 3 \theta+\frac{3}{4} \beta B_{41} \sin 3 \theta\right)\right]+h_{15}\left[\frac{1}{2} r^{-\frac{1}{2}} C_{11} .\right. \\
& \cos \frac{\theta}{2}-C_{21} \sin \theta+r^{\frac{1}{2}}\left(\frac{3}{2} C_{31} \cos \frac{3 \theta}{2}+\frac{1}{8} \beta C_{11} .\right. \\
& \left.\sin \frac{3 \theta}{2}+\frac{1}{8} \beta C_{11} \sin \frac{\theta}{2}\right)-r C_{41} \sin 2 \theta+r^{\frac{3}{2}}\left(\frac{5}{2} C_{51} .\right. \\
& \cos \frac{5 \theta}{2}-\frac{1}{8} \beta C_{31} \sin \frac{5 \theta}{2}+\frac{3}{64} \beta^{2} C_{11} \cos \frac{3 \theta}{2}+ \\
& \left.\frac{1}{96} \beta^{2} C_{11} \cos \frac{\theta}{2}+\frac{1}{8} \beta C_{31} \sin \frac{\theta}{2}\right)-r^{2}\left(3 C_{61} \sin 3 \theta+\right. \\
& \left.\left.\frac{1}{4} \beta C_{41} \cos 3 \theta+\frac{3}{4} \beta C_{41} \sin 3 \theta\right)\right]
\end{aligned}
$$$$
D_{r}=e_{15}\left[\frac{1}{2} r^{-\frac{1}{2}} A_{11} \sin \frac{\theta}{2}+A_{21} \cos \theta+\frac{3}{2} r^{\frac{1}{2}}\left(A_{31} \sin \frac{3 \theta}{2}-\right.\right.
$$$$
\left.\frac{1}{12} \beta A_{11} \cos \frac{3 \theta}{2}-\frac{1}{4} \beta A_{11} \cos \frac{\theta}{2}\right)+2 r A_{41} \cos 2 \theta+
$$$$
\frac{5}{2} r^{\frac{3}{2}}\left(A_{51} \sin \frac{5 \theta}{2}+\frac{1}{20} \beta A_{31} \cos \frac{5 \theta}{2}+\frac{1}{32} \beta^{2} A_{11} \sin \frac{3 \theta}{2}+\right.
$$$$
\left.\frac{1}{48} \beta^{2} A_{11} \sin \frac{\theta}{2}-\frac{1}{4} \beta A_{31} \cos \frac{\theta}{2}\right)+3 r^{2}\left(A_{61} \cos 3 \theta-\right.
$$$$
\left.\left.\frac{1}{12} \beta A_{41} \sin 3 \theta+\frac{1}{4} \beta A_{41} \cos 3 \theta\right)\right]-\varepsilon_{11}\left[\frac{1}{2} r^{-\frac{1}{2}} B_{11} \sin \frac{\theta}{2}+\right.
$$$$
B_{21} \cos \theta+\frac{3}{2} r^{\frac{1}{2}}\left(B_{31} \sin \frac{3 \theta}{2}-\frac{1}{12} \beta B_{11} \cos \frac{3 \theta}{2}-\frac{1}{4} \beta\right. \text {. }
$$$$
\left.B_{11} \cos \frac{\theta}{2}\right)+2 r B_{41} \cos 2 \theta+\frac{5}{2} r^{\frac{3}{2}}\left(B_{51} \sin \frac{5 \theta}{2}+\frac{1}{20} \beta\right. \text {. }
$$$$
B_{31} \cos \frac{5 \theta}{2}+\frac{1}{32} \beta^{2} B_{11} \sin \frac{3 \theta}{2}+\frac{1}{48} \beta^{2} B_{11} \sin \frac{\theta}{2}-
$$$$
\left.\frac{1}{4} \beta B_{31} \cos \frac{\theta}{2}\right)+3 r^{2}\left(B_{61} \cos 3 \theta-\frac{1}{12} \beta B_{41} \sin 3 \theta+\right.
$$$$
\left.\left.\frac{1}{4} \beta B_{41} \cos 3 \theta\right)\right]-d_{11}\left[\frac{1}{2} r^{-\frac{1}{2}} C_{11} \sin \frac{\theta}{2}+C_{21} \cos \theta+\right.
$$$$
\frac{3}{2} r^{\frac{1}{2}}\left(C_{31} \sin \frac{3 \theta}{2}-\frac{1}{12} \beta C_{11} \cos \frac{3 \theta}{2}-\frac{1}{4} \beta C_{11} \cos \frac{\theta}{2}\right)+
$$$$
2 r C_{41} \cos 2 \theta+\frac{5}{2} r^{\frac{3}{2}}\left(C_{51} \sin \frac{5 \theta}{2}+\frac{1}{20} \beta C_{31} \cos \frac{5 \theta}{2}+\right.
$$$$
\left.\frac{1}{32} \beta^{2} C_{11} \sin \frac{3 \theta}{2}+\frac{1}{48} \beta^{2} C_{11} \sin \frac{\theta}{2}-\frac{1}{4} \beta C_{31} \cos \frac{\theta}{2}\right)+
$$$$
\left.3 r^{2}\left(C_{61} \cos 3 \theta-\frac{1}{12} \beta C_{41} \sin 3 \theta+\frac{1}{4} \beta C_{41} \cos 3 \theta\right)\right]
$$$$
D_{\theta}=e_{15}\left[\frac{1}{2} r^{-\frac{1}{2}} A_{11} \cos \frac{\theta}{2}-A_{21} \sin \theta+r^{\frac{1}{2}}\left(\frac{3}{2} A_{31} \cos \frac{3 \theta}{2}+\right.\right.
$$$$
\left.\frac{1}{8} \beta A_{11} \sin \frac{3 \theta}{2}+\frac{1}{8} \beta A_{11} \sin \frac{\theta}{2}\right)-r A_{41} \sin 2 \theta+r^{\frac{3}{2}} \text {. }
$$ 


$$
\begin{aligned}
& \left(\frac{5}{2} A_{51} \cos \frac{5 \theta}{2}-\frac{1}{8} \beta A_{31} \sin \frac{5 \theta}{2}+\frac{3}{64} \beta^{2} A_{11} \cos \frac{3 \theta}{2}+\right. \\
& \left.\frac{1}{96} \beta^{2} A_{11} \cos \frac{\theta}{2}+\frac{1}{8} \beta A_{31} \sin \frac{\theta}{2}\right)-r^{2}\left(3 A_{61} \sin 3 \theta+\right. \\
& \left.\left.\frac{1}{4} \beta A_{41} \cos 3 \theta+\frac{3}{4} \beta A_{41} \sin 3 \theta\right)\right]-\varepsilon_{11}\left[\frac{1}{2} r^{-\frac{1}{2}} B_{11} .\right. \\
& \cos \frac{\theta}{2}-B_{21} \sin \theta+r^{\frac{1}{2}}\left(\frac{3}{2} B_{31} \cos \frac{3 \theta}{2}+\frac{1}{8} \beta B_{11} .\right. \\
& \left.\sin \frac{3 \theta}{2}+\frac{1}{8} \beta B_{11} \sin \frac{\theta}{2}\right)-r B_{41} \sin 2 \theta+r^{\frac{3}{2}}\left(\frac{5}{2} B_{51} .\right. \\
& \cos \frac{5 \theta}{2}-\frac{1}{8} \beta B_{31} \sin \frac{5 \theta}{2}+\frac{3}{64} \beta^{2} B_{11} \cos \frac{3 \theta}{2}+ \\
& \left.\frac{1}{96} \beta^{2} B_{11} \cos \frac{\theta}{2}+\frac{1}{8} \beta B_{31} \sin \frac{\theta}{2}\right)-r^{2}\left(3 B_{61} \sin 3 \theta+\right. \\
& \left.\left.\frac{1}{4} \beta B_{41} \cos 3 \theta+\frac{3}{4} \beta B_{41} \sin 3 \theta\right)\right]-d_{11}\left[\frac{1}{2} r^{-\frac{1}{2}} C_{11} .\right. \\
& \cos \frac{\theta}{2}-C_{21} \sin \theta+r^{\frac{1}{2}}\left(\frac{3}{2} C_{31} \cos \frac{3 \theta}{2}+\frac{1}{8} \beta C_{11} .\right. \\
& \left.\sin \frac{3 \theta}{2}+\frac{1}{8} \beta C_{11} \sin \frac{\theta}{2}\right)-r C_{41} \sin 2 \theta+r^{\frac{3}{2}}\left(\frac{5}{2} C_{51} .\right. \\
& \cos \frac{5 \theta}{2}-\frac{1}{8} \beta C_{31} \sin \frac{5 \theta}{2}+\frac{3}{64} \beta^{2} C_{11} \cos \frac{3 \theta}{2}+ \\
& \left.\frac{1}{96} \beta^{2} C_{11} \cos \frac{\theta}{2}+\frac{1}{8} \beta C_{31} \sin \frac{\theta}{2}\right)-r^{2}\left(3 C_{61} \sin 3 \theta+\right. \\
& \left.\left.\frac{1}{4} \beta C_{41} \cos 3 \theta+\frac{3}{4} \beta C_{41} \sin 3 \theta\right)\right]
\end{aligned}
$$

$$
\begin{aligned}
& H_{r}=h_{15}\left[\frac{1}{2} r^{-\frac{1}{2}} A_{11} \sin \frac{\theta}{2}+A_{21} \cos \theta+\frac{3}{2} r^{\frac{1}{2}}\left(A_{31} \sin \frac{3 \theta}{2}-\right.\right. \\
& \left.\frac{1}{12} \beta A_{11} \cos \frac{3 \theta}{2}-\frac{1}{4} \beta A_{11} \cos \frac{\theta}{2}\right)+2 r A_{41} \cos 2 \theta+ \\
& \frac{5}{2} r^{\frac{3}{2}}\left(A_{51} \sin \frac{5 \theta}{2}+\frac{1}{20} \beta A_{31} \cos \frac{5 \theta}{2}+\frac{1}{32} \beta^{2} A_{11} \sin \frac{3 \theta}{2}+\right. \\
& \left.\frac{1}{48} \beta^{2} A_{11} \sin \frac{\theta}{2}-\frac{1}{4} \beta A_{31} \cos \frac{\theta}{2}\right)+3 r^{2}\left(A_{61} \cos 3 \theta-\right. \\
& \left.\left.\frac{1}{12} \beta A_{41} \sin 3 \theta+\frac{1}{4} \beta A_{41} \cos 3 \theta\right)\right]-d_{11}\left[\frac{1}{2} r^{-\frac{1}{2}} B_{11} \sin \frac{\theta}{2}+\right. \\
& B_{21} \cos \theta+\frac{3}{2} r^{\frac{1}{2}}\left(B_{31} \sin \frac{3 \theta}{2}-\frac{1}{12} \beta B_{11} \cos \frac{3 \theta}{2}-\frac{1}{4} \beta .\right. \\
& \left.B_{11} \cos \frac{\theta}{2}\right)+2 r B_{41} \cos 2 \theta+\frac{5}{2} r^{\frac{3}{2}}\left(B_{51} \sin \frac{5 \theta}{2}+\frac{1}{20} \beta\right. \text {. } \\
& B_{31} \cos \frac{5 \theta}{2}+\frac{1}{32} \beta^{2} B_{11} \sin \frac{3 \theta}{2}+\frac{1}{48} \beta^{2} B_{11} \sin \frac{\theta}{2}- \\
& \left.\frac{1}{4} \beta B_{31} \cos \frac{\theta}{2}\right)+3 r^{2}\left(B_{61} \cos 3 \theta-\frac{1}{12} \beta B_{41} \sin 3 \theta+\right. \\
& \left.\left.\frac{1}{4} \beta B_{41} \cos 3 \theta\right)\right]-\mu_{11}\left[\frac{1}{2} r^{-\frac{1}{2}} C_{11} \sin \frac{\theta}{2}+C_{21} \cos \theta+\right. \\
& \frac{3}{2} r^{\frac{1}{2}}\left(C_{31} \sin \frac{3 \theta}{2}-\frac{1}{12} \beta C_{11} \cos \frac{3 \theta}{2}-\frac{1}{4} \beta C_{11} \cos \frac{\theta}{2}\right)+ \\
& 2 r C_{41} \cos 2 \theta+\frac{5}{2} r^{\frac{3}{2}}\left(C_{51} \sin \frac{5 \theta}{2}+\frac{1}{20} \beta C_{31} \cos \frac{5 \theta}{2}+\right. \\
& \left.\frac{1}{32} \beta^{2} C_{11} \sin \frac{3 \theta}{2}+\frac{1}{48} \beta^{2} C_{11} \sin \frac{\theta}{2}-\frac{1}{4} \beta C_{31} \cos \frac{\theta}{2}\right)+ \\
& \left.3 r^{2}\left(C_{61} \cos 3 \theta-\frac{1}{12} \beta C_{41} \sin 3 \theta+\frac{1}{4} \beta C_{41} \cos 3 \theta\right)\right]
\end{aligned}
$$




$$
\begin{aligned}
& H_{\theta}=h_{15}\left[\frac{1}{2} r^{-\frac{1}{2}} A_{11} \cos \frac{\theta}{2}-A_{21} \sin \theta+r^{\frac{1}{2}}\left(\frac{3}{2} A_{31} \cos \frac{3 \theta}{2}+\right.\right. \\
& \left.\frac{1}{8} \beta A_{11} \sin \frac{3 \theta}{2}+\frac{1}{8} \beta A_{11} \sin \frac{\theta}{2}\right)-r A_{41} \sin 2 \theta+r^{\frac{3}{2}} \text {. } \\
& \left(\frac{5}{2} A_{51} \cos \frac{5 \theta}{2}-\frac{1}{8} \beta A_{31} \sin \frac{5 \theta}{2}+\frac{3}{64} \beta^{2} A_{11} \cos \frac{3 \theta}{2}+\right. \\
& \left.\frac{1}{96} \beta^{2} A_{11} \cos \frac{\theta}{2}+\frac{1}{8} \beta A_{31} \sin \frac{\theta}{2}\right)-r^{2}\left(3 A_{61} \sin 3 \theta+\right. \\
& \left.\left.\frac{1}{4} \beta A_{41} \cos 3 \theta+\frac{3}{4} \beta A_{41} \sin 3 \theta\right)\right]-d_{11}\left[\frac{1}{2} r^{-\frac{1}{2}} B_{11}\right. \text {. } \\
& \cos \frac{\theta}{2}-B_{21} \sin \theta+r^{\frac{1}{2}}\left(\frac{3}{2} B_{31} \cos \frac{3 \theta}{2}+\frac{1}{8} \beta B_{11}\right. \text {. } \\
& \left.\sin \frac{3 \theta}{2}+\frac{1}{8} \beta B_{11} \sin \frac{\theta}{2}\right)-r B_{41} \sin 2 \theta+r^{\frac{3}{2}}\left(\frac{5}{2} B_{51} .\right. \\
& \cos \frac{5 \theta}{2}-\frac{1}{8} \beta B_{31} \sin \frac{5 \theta}{2}+\frac{3}{64} \beta^{2} B_{11} \cos \frac{3 \theta}{2}+ \\
& \left.\frac{1}{96} \beta^{2} B_{11} \cos \frac{\theta}{2}+\frac{1}{8} \beta B_{31} \sin \frac{\theta}{2}\right)-r^{2}\left(3 B_{61} \sin 3 \theta+\right. \\
& \left.\left.\frac{1}{4} \beta B_{41} \cos 3 \theta+\frac{3}{4} \beta B_{41} \sin 3 \theta\right)\right]-\mu_{11}\left[\frac{1}{2} r{ }^{-\frac{1}{2}} C_{11}\right. \text {. } \\
& \cos \frac{\theta}{2}-C_{21} \sin \theta+r^{\frac{1}{2}}\left(\frac{3}{2} C_{31} \cos \frac{3 \theta}{2}+\frac{1}{8} \beta C_{11}\right. \text {. } \\
& \left.\sin \frac{3 \theta}{2}+\frac{1}{8} \beta C_{11} \sin \frac{\theta}{2}\right)-r C_{41} \sin 2 \theta+r^{\frac{3}{2}}\left(\frac{5}{2} C_{51}\right. \text {. } \\
& \cos \frac{5 \theta}{2}-\frac{1}{8} \beta C_{31} \sin \frac{5 \theta}{2}+\frac{3}{64} \beta^{2} C_{11} \cos \frac{3 \theta}{2}+ \\
& \left.\frac{1}{96} \beta^{2} C_{11} \cos \frac{\theta}{2}+\frac{1}{8} \beta C_{31} \sin \frac{\theta}{2}\right)-r^{2}\left(3 C_{61} \sin 3 \theta+\right. \\
& \left.\left.\frac{1}{4} \beta C_{41} \cos 3 \theta+\frac{3}{4} \beta C_{41} \sin 3 \theta\right)\right]
\end{aligned}
$$

The mode III stress intensity factor (SIF) of the crack tip are defined as

$$
\left\{\begin{array}{l}
K^{T}=\lim _{r \rightarrow 0} \sqrt{2 \pi r} \tau_{\theta z}(r, 0)=\frac{\sqrt{2 \pi}}{2}\left(c_{440} A_{11}+e_{150} B_{11}+h_{150} C_{11}\right) \\
K^{D}=\lim _{r \rightarrow 0} \sqrt{2 \pi r} D_{\theta}(r, 0)=\frac{\sqrt{2 \pi}}{2}\left(e_{150} A_{11}-\varepsilon_{110} B_{11}-d_{110} C_{11}\right) \\
K^{B}=\lim _{r \rightarrow 0} \sqrt{2 \pi r} B(r, 0)=\frac{\sqrt{2 \pi}}{2}\left(h_{150} A_{11}-d_{110} B_{11}-\mu_{110} C_{11}\right)
\end{array}\right.
$$

\section{CONCLUSION}

The crack-tip higher order fields of magnetoelectroelastic materials are obtained by the eigen-expansion method in this paper. The first two items of crack-tip higher order fields have the same mathematical form as ones of homogeneous materials. The effect of nonhomogeneity reflects only in the higher order items. Due to coupling effect of magnetoelectroelastic material, crack-tip higher order fields are depending on displacement component, the electric potential and magnetic potential. The crack tip higher-order fields are equivalent to the eigen-functions mathematically. Therefore, they provided the theoretical basis for the numerical simulations, engineering applications, experimental analysis of crack problems of magnetoelectroelastic materials

\section{ACKNOWLEDGMENT}

The research is supported by the National Natural Science Foundation of China (No11172332.).

\section{REFERENCES}

[1] Wang B L, Han J C, Mai Y W, "Mode Iii Fracture of a Magnetoelectroelastic Layer: Exact Solution and Discussion of the Crack Face Electromagnetic Boundary Conditions," International Journal of Fracture, vol.139, 2006, pp. 27-38, doi:10.1007/s10704006-6632-1.

[2] Wan Y, Yue Y, Zhong Z, "A Mode III Crack Crossing the Magnetoelectroelastic Bimaterial Interface under Concentrated Magnetoelectromechanical Loads," International Journal of Solids and Structures, vol.49, 2012, pp. 3008-3021, doi:10.1016/j.ijsolstr.2012.06.001.

[3] Zhong X-C, Li X-F, "Magnetoelectroelastic Analysis for an Opening Crack in a Piezoelectromagnetic Solid," European Journal of Mechanics - A/Solids, vol.26, 2007, pp. 405-417, doi:10.1016/j.euromechsol.2006.08.002.

[4] Huang G Y, Wang B L, Mai Y W, "Effect of Interfacial Cracks on the Effective Properties of Magnetoelectroelastic Composites," Journal of Intelligent Material Systems and Structures, vol.20, 2009, pp. 963-968, doi:10.1177/1045389x08101564.

[5] Gao C-F, Kessler H, Balke H, "Crack Problems in Magnetoelectroelastic Solids. Part Ii: General Solution of Collinear Cracks," International Journal of Engineering Science, vol.41, 2003, pp. 983-994, doi: 10.1016/S0020-7225(02)00324-5.

[6] Sih G C, Jones R, Song Z F, "Piezomagnetic and Piezoelectric Poling Effects on Mode I and Ii Crack Initiation Behavior of Magnetoelectroelastic Materials," Theoretical and Applied Fracture Mechanics, vol.40, 2003, pp. 161-186, doi:10.1016/s01678442(03)00044-2.

[7] Li Y-D, Lee K Y, "Fracture Analysis on a Piezoelectric Sensor with a Viscoelastic Interface," European Journal of Mechanics, vol.28, 2009, pp. 738-743, doi:10.1016/j.euromechsol.2009.02.003.

[8] Athanasius L, Ang W T, "Magnetoelectroelastodynamic Interaction of Multiple Arbitrarily Oriented Planar Cracks," Applied Mathematical Modelling2013, pp. doi: 10.1016/j.apm.2013.02.013.

[9] Bhargava R R, Sharma K, "Application of X-Fem to Study TwoUnequal-Collinear Cracks in 2-D Finite Magnetoelectoelastic Specimen," Computational Materials Science, vol.60, 2012, pp. 75-98, doi: 10.1016/j.commatsci.2012.03.013.

[10] Tupholme G E, "Magnetoelectroelastic Media Containing a Row of Moving Shear Cracks," Mechanics Research Communications, vol.45, 2012, pp. 48-53, doi: 10.1016/j.mechrescom.2012.07.002.

[11] Wang B L, Mai Y W, "Crack Tip Field in Piezoelectric/Piezomagnetic Media," European Journal of Mechanics a-Solids, vol.22, 2003, pp. 591-602, doi:10.1016/s09977538(03)0062-7.

[12] Zheng J L, Fang Q H, Liu Y W, “A Generalized Screw Dislocation Interacting with Interfacial Cracks Along a Circular Inhomogeneity in Magnetoelectroelastic Solids," Theoretical and Applied Fracture Mechanics, vol.47, 2007, pp. 205-218, doi:10.1016/j.tafmec.2007.01.005.

[13] Ma P, Feng W J, Su R K L, “An Electrically Impermeable and Magnetically Permeable Interface Crack with a Contact Zone in a Magnetoelectroelastic Bimaterial under Uniform Magnetoelectromechanical Loads," European Journal of Mechanics A/Solids, vol.32, 2012, pp. 41-51, doi: 10.1016/j.euromechsol.2011.09.010.

[14] Li X F, Liu G L, Lee K Y, "Magnetoelectroelastic Field Induced by a Crack Terminating at the Interface of a Bi-Magnetoelectric Material," Philosophical Magazine, vol.89, 2009, pp. 449-463, doi:10.1080/14786430802653428. 